\title{
Structural health monitoring through surface acoustic wave inspection deployed on capillaries embedded in additively manufactured components
}

\author{
Michaël Hinderdael ${ }^{1}$, Zoé Jardon ${ }^{1}$, Julien Ertveldt ${ }^{1}$, Patrick Guillaume ${ }^{1}$ \\ ${ }^{1}$ Department of Mechanical Engineering, Vrije Universiteit Brussel, Belgium
}

\begin{abstract}
Surface Acoustic Wave inspection is a well-known nondestructive testing technique that receives considerable attention to become implemented as a Structural Health Monitoring system. The current work presents a novel approach to embed Surface Acoustic Wave-based Structural Health Monitoring technology inside additively manufactured components. A capillary network is to be integrated inside the component and Surface Acoustic Wave inspection is then deployed on the free capillary surface during the component's operation to warn upcoming failures.
\end{abstract}

\section{Introduction}

Surface Acoustic Waves (SAW) or Rayleigh Waves are acoustic waves that propagate along the free surface of a material. The SAWs exhibit elastic motion and contain components of displacement normal to the surface. Most of the surface acoustic energy is contained in the Rayleigh pulse, which propagates at a constant velocity $v_{R}$, independent of the frequency. Their amplitude typically decays exponentially into material with most energy concentrated in a one-wavelength thick waveguide just below the free surface $[1,2]$.

Besides various other applications where SAW are put to great use, the analysis of these waves also serves as a useful tool in non-destructive characterization of materials. With a shallow penetration depth that decreases with increasing frequency, SAWs are very sensitive to (sub)surface imperfections, such as scratches, cracks, voids, etc. These surface imperfections cause both dispersion, i.e. dependency of the phase velocity to the wave frequency and attenuation, providing a fingerprint of the inspected surface [2].

SAWs have the unique ability to confine themselves inside the thin-wall structures and can therefore generally propagate along large distances without significant attenuation, making them suitable to inspect very large structures (e.g. pipelines). SAWs have been used inside curved structures and have successfully detected and localized defects, as well as corrosion under insulation (CUI), in pipelines and various metallic and non-metallic structures [3-5]. The high sensitivity to surface/subsurface flaws, long reach, efficiency in finding faults of inaccessible areas have made this approach a cost-effective and preferable choice for structural health assesment $[3,6-7]$. 
Despite the many benefits of the technology, still many challenges remain. In their recent review work, Abbas and Shafiee highlighted the impact of the surface condition on which the SAWs propagate. The authors reported wetting/icing of external surfaces, exterior acoustic signals and surface protective measures as significant challenges that may not disturb the propagating waves [3]. All surface interactions influence the signature of the SAW inspection, potentially disturbing the SHM analysis.

The current work presents a novel approach to deploy SAW inspection as an embedded SHM technology, particularly suited to be produced by means of additive manufacturing (AM). Purposedly created channels inside the component serve as the SAW waveguide for the inspection, allowing the analysis to be redirected to particular areas of interest. Additionally, robustness is improved by embedding the SAW inspection technology into the component, preventing detrimental surface alterations to occur.

The remainder of this manuscript will present the concept in more detail, describe the initial sensor design, demonstrate the production of the embedded channels and report the initial proof-of-concept results.

\section{Embedded SHM by AM}

\subsection{State of the art}

Additive Manufacturing (AM) is a relatively new manufacturing technology that, as opposed to subtractive technologies, creates objects from scratch in a layerwise manner. Based on 3D model data, loose powder particles are selectively joined together, in order to create the desired component. As the production process evolves layer by layer, additional functional features - such as the forementioned channels - can be integrated during the production process. Design optimization for weight reduction typically drives towards very complex shaped components that can now be produced by AM. Inspection of those components will require dedicated inspection technology such as an embedded SHM solution.

The use of embedded channels for SHM purposes, also referred to as the eSHM system, has been presented in the literature before. Hinderdael et al. utilized the capability to pressurize the channels inside the AM components to measure deformation and component's usage $[8,9]$. De Baere et al. presented an SHM technology based on the same pressurization principle [10]. Initial proof-of-concept studies of successful fatigue crack detection were presented on specimens produced by means of laser-based Powder Bed Fusion (L-PBF) $[11,12]$ and laser-based Directed Energy Deposition [11,13,14-16] with particular focus on capillary topology [14], surface roughness [12,15,16] and material (Ti-6Al-4V[11,12,14,15] and SS316L[11,13]). An example of a complex shaped channel, embedded by means of AM, has been depicted in Fig. 1.

Hinderdael et al. [17] presented a crack localization principle based on the time-of-flight difference of pressure waves propagating in the fluid media inside the integrated capillaries. This principle was further researched $[18,19]$ but to date no successful crack localization has been achieved during an actual fatigue crack event. De Pauw et al. post-inserted an optical fibre through the additively created channels and utilized distributed strain sensing to successfully detect and localize fatigue cracks [20]. 


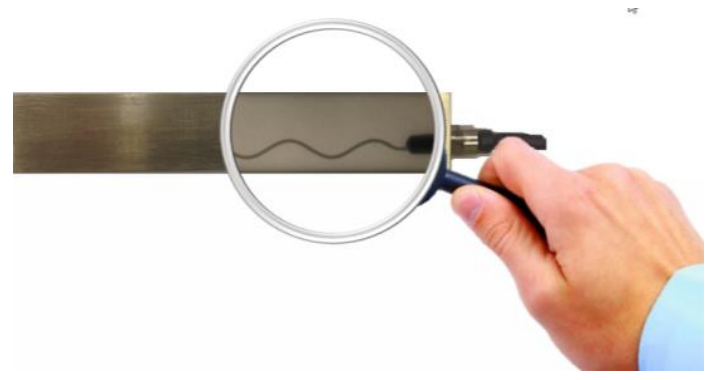

Fig. 1. Example of a complex shaped channel embedded by means of AM technology for SHM purposes. Inset is an X-RAY photograph.

\subsection{Surface Acoustic Waves on Capillary Surface}

A new SHM concept utilizes the same capillary for SAW inspection in which case the capillary acts as the free surface for SAW propagation. SAWs on the capillary surface will propagate along the capillary length and react to (surface) imperfections in a shallow band around the capillary. As the capillary now acts as a one-dimensional waveguide with small dimensions, the SAWs are expected to propagate with higher amplitudes and even less attenuation than their 2-dimensional counterparts on thin plate surfaces. The proposed SHM methodology is expected to be reliable and robust as undesired surface interactions are avoided by embedding the technology.
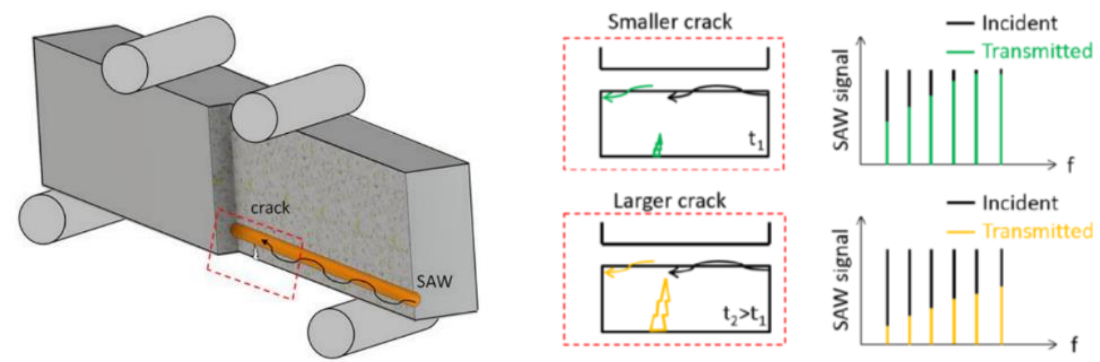

Fig. 2. Sketch of SAW interaction with fatigue crack during four-point bending fatigue testing.

The SAWs are expected to reflect, and limit transmission, as soon as (fatigue) cracks or other potential material imperfections interact with the SAWs. This principle is depicted in Fig. 2. The inspection depth around the circumference of the channel (highlighted with a red dashed square) is depending on the chosen frequency. For lower frequencies (larger wavelengths), larger fractions of the material can be inspected. Crack size may be quantified by varying testing frequencies and evaluating signal attenuation w.r.t. the initial state prior to usage. Crack propagation may be followed up using the same principle or by evaluating further signal attenuation at a single inspection frequency. 


\section{Development of a SAW excitation solution for capillaries}

The current state-of-art allows the integration of $1 \mathrm{~mm}$ diameter capillaries by means of LPBF and $2 \mathrm{~mm}$ in diameter by means of L-DED technology. Further reducing the crosssectional dimensions of the channel is clearly driven by a further reduction of their structural impact on the component while it also further focuses the SAW energy on the smaller capillary circumference. However, the miniaturization of the technology poses additional challenges that are not considered part of this work.

The Olympus V542-SM videoscan angle beam transducers were used as basis for the current work and had a nominal element size of $6 \mathrm{~mm}$, which lead to the decision to conduct early proof-of-concept studies to $6 \mathrm{~mm}$ diameter capillaries. The transducers have a rated $2.25 \mathrm{MHz}$ centre frequency but presented an optimal frequency for maximized signal transmittance (without any intermediate medium) at $1.56 \mathrm{MHz}$. The transducer is combined with a Perspex wedge to convert the longitudinal mode into a Rayleigh wave on the Aluminum-7075 surface of interest. Table 2. presents the acoustic properties of the materials involved that served for the design of the wedge at three inspection frequencies (1, 1.56 and $2 \mathrm{MHz}$ ).

Table 2. Acoustic properties of the materials involved at various inspection frequencies.

\begin{tabular}{|c|c|c|c|c|}
\hline & Longitudinal Wave & \multicolumn{3}{|c|}{ Rayleigh Wave } \\
\cline { 2 - 5 } & $\boldsymbol{v}_{L}[\mathbf{m} / \mathbf{s}]$ & $\boldsymbol{f}_{c}[\mathbf{M H z}]$ & $\boldsymbol{v}_{R}[\mathbf{m} / \mathbf{s}]$ & $\lambda_{R}[\mathbf{m m}]$ \\
\hline Perspex/Plexi & $2730[21]$ & & & \\
\hline \multirow{3}{*}{ Aluminum 7075 } & & 1.00 & \multirow{2}{*}{$2888[22]$} & 2.88 \\
\cline { 3 - 3 } & & 1.56 & & 1.85 \\
\cline { 3 - 5 } & & 2.00 & & 1.44 \\
\hline
\end{tabular}

The current analysis will focus on a half-open capillary milled in an Aluminum 7075 alloy base material. Such open capillary is an intermediate step towards fully enclosed capillaries that now still provides ease-of-access to relocate the transducers and mimic surface damages. As no wedge exists off-the-shelve to excite Rayleigh waves on such miniaturized tubing/capillaries, a wedge with a semi-circular contact surface was manufactured.The wedge design was based on a ABWML-4T $-90^{\circ}$ wedge with an angle equal to the critical angle of incidence $\left(\theta_{R}\right)$ for the generation of Rayleigh waves on the Aluminum 7075 substrate. Snell's Law is expressed as:

$$
\theta_{R}=\sin ^{-1}\left(\frac{v_{L}}{v_{R}}\right)=71^{\circ}
$$
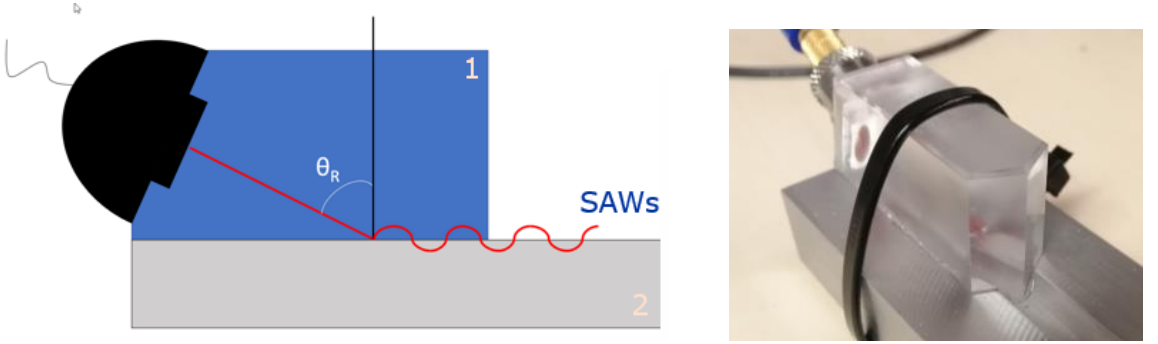

Fig. 3. Angled wedge transducer with a $6 \mathrm{~mm}$ diameter semi-circular excitation surface. 


\section{Experimental setup \& results}

The experimental setup is comprised by two angle beam transducers located at the very ends of an Aluminum 7075 alloy beam $(300 \times 40 \times 15 \mathrm{~mm})$ and fit inside the $6 \mathrm{~mm}$ semi-circular engraved channel. The contact surfaces between the transducers and the wedges on the one hand, and the wedges with the channel on the other hand, are wetted using glycerine as ensure maximal signal transmittance. The wedge is designed to ensure contact with the channel's surface but avoiding contact with the flat top surface of the beam.

The excitation signals were either created using an Agilent 33210A function generator was used to create a continuous sinusoidal signal with an amplitude of $3 \mathrm{Vpp}$. This signal was both fed to a first channel of the National Instruments PXI1033 DAQ unit and to a Falco Systems WMA-300 High voltage amplifier (x50) rated to output 150Vpp at maximum. This signal was then sent to the Olympus V542-SM transducer (transmitter) and received by a similar second transducer (pitch-catch mode, transmission mode). The converted electrical signal was then fed to a second channel on the same DAQ system. A labview v14 script was used to control the DAQ and post processing of the data was conducted in Matlab v2021a.

To replicate the gradual growth of a single fatigue crack, successive sawing operations were executed to the bottom side of the specimen under investigation. The simulated crack had successive depths of 5,7,9,11,12 and finally $13 \mathrm{~mm}$. With a beam thickness of $15 \mathrm{~mm}$ and a semi-circular capillary of $3 \mathrm{~mm}$ radius, a simulated crack that propagated $12 \mathrm{~mm}$ from the bottom up will exactly meet the capillary surface. The different sawing depths therefore represent cracks that are separated to the capillary by a remaining distance of 7,5,3,1,0 and $1 \mathrm{~mm}$. In between each sawing operation, SAWs were subsequently excited on the capillary surface at three different frequencies $(1,1.56$ and $2 \mathrm{MHz})$ and the response at the receiver side was monitored, representing the transmitted SAW signal. Fig. 5. presents the average amplitude (and their standard deviation) of 20 successive sines as received by the receiving transducer.

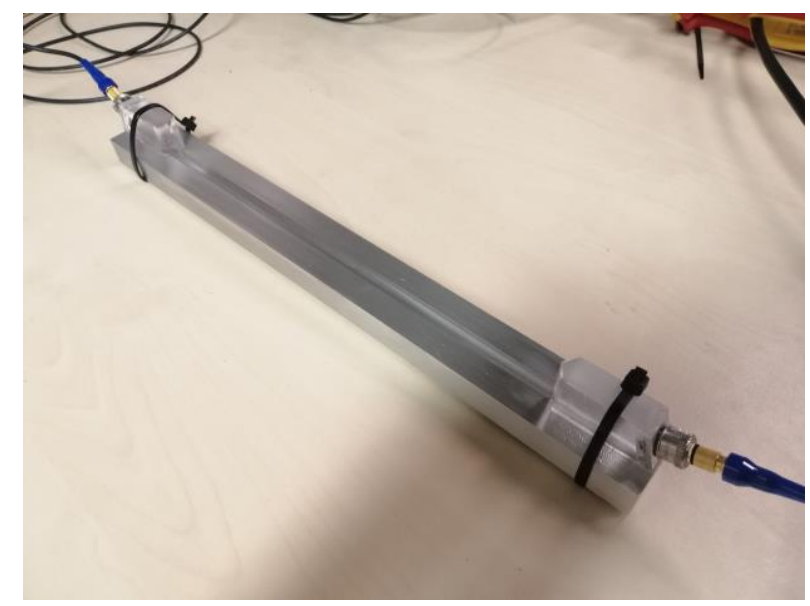

Fig. 4. Transitter and receiver interfacing a semi-circular channel to mimic the embedded Structural Health Monitoring system based on SAW inspection. 


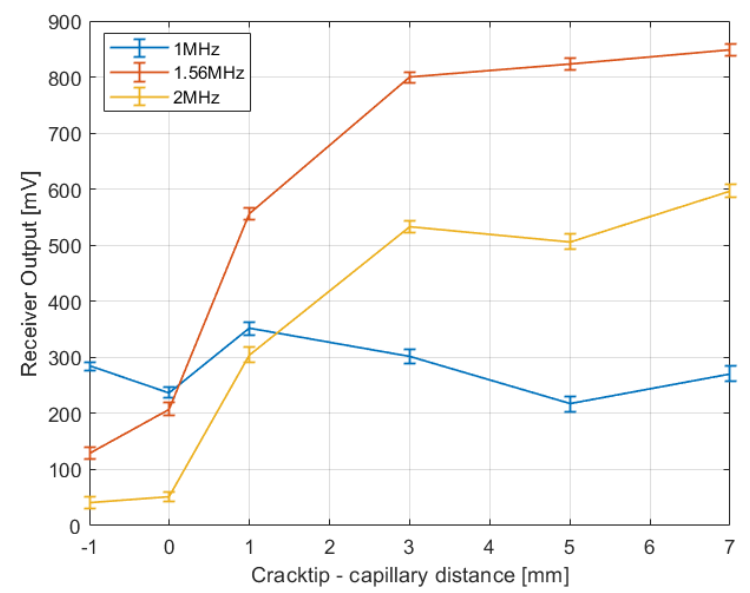

Fig. 5. Receiver output at various inspection frequencies at different crack sizes.

From Fig. 5. it may be noticed that no loss of signal is observed when the crack is still separated by approximately $3 \mathrm{~mm}$, which confirms the shallow depth of SAW propagation that is not impacted by this damage. Indeed, with wavelengths varying from $1.44 \mathrm{~mm}$ to 2.88 $\mathrm{mm}$, it is not expected that the signal penetrates that deep into the material.

The next datapoint, at $1 \mathrm{~mm}$ separation between the fatigue crack and the channel, presents significant signal attenuation for the $1.56 \mathrm{MHz}$ and $2 \mathrm{MHz}$ inspection frequencies and allows concluding that the damage has been detected by the embedded SAW inspection method. Further growth of the damage further attenuates the transmitted signal. The $1 \mathrm{MHz}$ inspection frequency presented no obvious signal attenuation (and thus no damage detection), but signals were significantly weaker throughout the test and potentially impacting the inspection.

\section{Conclusions}

Additive Manufacturing (AM) allows embedding functional features inside the component. A novel principle for embedded SHM, based on SAW inspection, was presented in the current manuscript. Channels are to be integrated by means of additive manufacturing, of which their inner surfaces are then utilized as free surfaces for SAW propagation. The channels serve as acoustic waveguides, allowing to redirect the inspection to particular areas of interest for inspection. Various tests were conducted at three inspection frequencies $(1 \mathrm{MHz}, 1.56 \mathrm{MHz}$ and $2 \mathrm{MHz})$ on an Aluminum 7075 coupon which presented successively larger damages. This work presented the capability to excite SAW on capillary surfaces and confirmed the concept to detect damages by means of SAW inspection on embedded channels. Damages are detected as soon as they reach the penetration depth of the SAW, with presenting further signal attenuation at larger damage sizes. 


\section{Acknowledgements}

The research work has been financed by Research Foundation Flanders (FWO), grant nr. $12 Z V 920 N$.

\section{References}

[1] J. A. Cooper, R. A. Crosbie, R. J. Dewhurst, A. D. W. McKie, S. B. Palmer, IEEE Trans Ultrason Ferroelectr Freq Contro, Vol. UFFC-33, No.5, p. 462-470, (1996)

[2] A. Tarasenko, R. Ctvrtlik, R. Kudelka, Sci. Rep. , 11, 2845, (2021)

[3] M. Abbas, M. Shafiee, Structural health monitoring (shm) and determination of surface defects in large metallic structures using ultrasonic guided waves, Sensors (Basel), 18, 3958, p. 1-26, (2018)

[4] P. Wilcox, M. Lowe, P. Cawley, The effect of dispersion on long-range inspection using ultrasonic guided waves,NDT E Int, 34, p. 1-9, (2001)

[5] B. Zima, M. Rucka, Guided waves for monitoring of plate structures with linear cracks of variable length. Arch. Civ. Mech. Eng. 16, p. 387-396, (2016)

[6] J. L. Rose, Ultrasonic Guided Waves in Solid Media, 1st ed., Cambrdige University Press: New York, NY, USA, (2014) ISBN: 978-1-107-04895-9

[7] P. Rizzo, J. G. Han, X. L. Ni, Structural health monitoring of immersed structures by means of guided ultrasonic waves. J. Intell. Mater. Syst. Struct. 21, p. 3769-3775, (2010)

[8] M. Hinderdael, Z. Jardon, M. Lison, D. De Baere, W. Devesse, M. Strantza, P. Guillaume, Proof of concept of integrated load measurement in 3D printed structures, Sensors (Basel), 17, 2, 328, p. 1-12

[9] M. Hinderdael, M. Moonens, J. Ertveldt, D. De Baere, P. Guillaume, Analytical Modeling of Embedded Load Sensing Using Liquid-filled Capillaries Integrated by Metal Additive Manufacturing, IEEE Sens. J., 19, 20, p. 9447-9455, (2019)

[10] D. De Baere, M. Strantza, M. Hinderdael, W. Devesse, P. Guillaume, Effective Structural Health Monitoring with Additive Manufacturing, Proceedings Of 7th European Workshop on Structural Health Monitoring, p. 2314-2321 (2014)

[11] M. Strantza, D. De Baere, M. Rombouts, S. Clijsters, I. Vandendael, H. Terryn, P. Guillaume, D. Van Hemelrijck, 3D Printing for Intelligent Metallic Structures, Proceedings Of 7th European Workshop on Structural Health Monitoring, p. 2251 - 2257, (2014)

[12] M. F. Hinderdael, D. De Baere, P. Guillaume, Fatigue performance of powder bed fused Ti-6Al-4V component with integrated chemically etched capillary for structural health monitoring application, Proceedings of the 18th International Conference on Experimental Mechanics, 2, 8, 499, p. 1-5, (2018)

[13] M. Strantza, D. De Baere, M. Rombouts, G. Maes, P. Guillaume, D. Van Hemelrijck, Feasibility study on structural health monitoring system produced by metal threedimensional printing, Struct. Health Monit., 14, 6, (2015)

[14] M. Strantza, R. Vafadari, D. De Baere, M. Rombouts, I. Vandendael, H. Terryn, M. Hinderdael, A. Rezaei, W. Van Paepeghem, P. Guillaume, D. Van Hemelrijck, Evaluation of different topologies of integrated capillaries in effective structural health monitoring system produced by 3D printing, Proceedings of 10th International Workshop on Structural Health Monitoring, 1, p. 153-160 (2015)

[15] M. Strantza, M. Hinderdael, D. De Baere, I. Vandendael, H. Terryn, D. Van Hemelrijck, P. Guillaume, Additive Manufacturing for novel structural health monitoring 
systems, proceedings of the 8th European Workshop on Structural Health Monitoring, 367, p. 1-9 (2016)

[16] M. Hinderdael, M. Strantza, D. De Baere, W. Devesse, I. De Graeve, H. Terryn, P. Guillaume, Fatigue performance of Ti-6Al-4V additively manufactured specimens with integrated capillaries of an embedded structural health monitoring system, Materials, 10, 9, (2017)

[17] M. F. Hinderdael, D. De Baere, P. Guillaume, Proof of concept of Crack Localization Using Negative Pressure Waves in Closed Tubes for Later Application in Effective SHM system for Additive Manufactured Components, Appl. Sci., 6, 33, p. 1-12, (2016)

[18] M. Hinderdael, Z. Jardon, P. Guillaume, An analytical model for negative pressure waves in gaseous media, Mech Syst Signal Process, 144, 106800, (2020)

[19] Z. Jardon, M. Hinderdael, T. Regert, J. Van Beeck, P. Guillaume, On the nature of Pressure Wave Propagation through Ducts for Structural Health Monitoring Application, Appl. Sci., 9, 5, 837, p. 1-15, (2019)

[20] B. De Pauw, M. Hinderdael, M. Moonens, D. De Baere, T. Geernaert, F. Berghmans, P. Guillaume, Fatigue failure monitoring of $316 \mathrm{~L}$ stainless coupons using optical fibre based distributed strain sensing, Smart Mater Struct., 28, 10, (2019)

[21] Catalogus Olympus

[22] A. D. W. McKie, J. W. Wagner, J. B. Spicer, J. B. Deaton, Dual-beam interferometer for the accurate determination of surface-wave velocity. Applied Optics, 30, 28, pp. 40344039, (1991) 